\title{
Des génotypes rouillés...
}

\section{Estelle Cadet, Renaud Warin, Anne-Sophie Perez, Dominique Capron, Jacques R ochette}

II existe deux grands groupes de maladies gén étiques responsables d'une surcharge en fer - les thalassémies et les hémochromatoses - qui détiennent des records de prévalence mais n'affectent pas les mêmes populations. Si I'on porte sur un planisphère les zones de leur distribution respective, on constate que, là où les thalassémies sont absentes, I'hémochromatose est présente. De nombreuses en qu êtes permettent désormais d'établir les fréqu ences de ces différents allèles morbides en fonction des origines géographiques voire ethniques des sujets testés. Ainsi, en ce qui concerne la mutation C282Y du gène H FE-I (hémochromatose génétique), sa fréquence moyenne en Europe est de $6,6 \%$ et décroît sel on un gradient nord-ou est/ sud-est. Une pression de sélection pour les mutations thalassémiques par l'agent du palu disme a été proposée. Pour l'hémochromatose, il est peu probable que l'ampleur de la mutation causale soit la con séqu ence d'un phén omène de dérive génétique. L'âge récent de la mutation et sa fréquence élevée sont en défaveur d'une telle hypothèse. Nos résultats et ceux d'au tres équipes su ggèrent, comme pour les thalassémies, une forte pression de sélection des allèles mutés. Parmi les hypothèses en visagées, celle d'une résistance à un agent infectieux utilisant la proténe H FE comme récepteur paraît plausible, sans que l'on puisse éliminer toutefois un mode de sélection directe sur le métabolisme du fer.

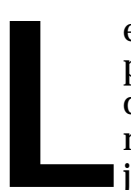

es constitutions phénotypiques des populations, qu'elles soient végétales, animales ou humaines ont toulours retenu l'attention de nombreux spécialistes des disciplines scientifiques ou médicales. La plus simple des observations biologiques, celle qui consiste à noter des caractères morphologiques, révèle une variabilité phénotypique impressionnante et a été le point de départ de l'étude des mutants. Cette variabilité macroscopique a été pendant très longtemps la seule source d'inspiration des généticiens.

Dans les années 1960, grâce aux techniques électrophorétiques appliquées à la séparation des protéines et à la révélation spécifique des activités enzymatiques, on est passé de l'étude des variabilités morphologiques à celle de la nature biochimique des polymorphismes et des mutations.
L'introduction des techniques de la génétique moléculaire dans les années 1970 a permis, entre autres, la mise en évidence rapide de polymorphismes et de mutations dans les séquences d'ADN. Un certain nombre de succès ont été alors enregistrés principalement dans le domaine du diagnostic biologique et de la physiopathologie des maladies génétiques. On a en effet retiré de l'étude des mutations responsables des maladies génétiques une certaine aptitude à déduire le potentiel pathogène de ces dernières et les notions d'hétérogénéité génétique ou de variation allélique ont été illustrées d'exemples précis.

Si l'on sait maintenant que la simple hétérogénéité allélique (plusieurs mutations différentes dans un gène donné, capables pour chacune d'elles d'entraîner la maladie) peut engendrer des différences phénoty- piques importantes, jusqu'au point de modifier la symptomatologie et la physiopathologie d'une maladie génétique, on sait aussi que des gènes mutés différents peuvent être à l'origine de modifications phénotypiques très voisines (hétérogénéité génétique) : c'est le cas notamment des anomalies qui affectent des gènes impliqués dans l'absorption, le transport ou la fixation du fer.

II existe en effet des maladies génétiques dont la prévalence est particulièrement élevée et dont une des particularités majeures est une atteinte tissulaire due à un excès de stockage du fer. Certaines de ces surcharges sont primaires et d'autres secondaires, chacune d'elles pouvant être due à des mutations affectant des gènes différents. Cette hétérogénéité de locus aboutit à une distribution géographique des surcharges en fer extrêmement large: sur tous les 
continents, des populations ethniquement bien différentes sont porteuses d'allèles mutés différents pour une surcharge en fer à une fréquence relativement élevée. Ces maladies sont principalement les hémochromatoses et les thalassémies, qui détiennent des records de prévalence.

\section{L'hémochromatose HFE-1}

Les surcharges en fer primaires sont essentiellement familiales et constituent le groupe des hémochromatoses dont la forme la plus fréquente, I'hémochromatose génétique (HFE-1) est transmise selon le mode autosomique récessif. Elle atteint entre 2 à 5 sujets sur mille chez les descendants des populations du N ord-O uest de l'Europe. Elle se constitue cliniquement par le biais d'une hyperabsorption digestive de fer qui aboutit à son accumulation tissulaire excessive, surtout responsable d'une atteinte hépatique. Son expression est tardive, l'âge moyen du diagnostic se situant rarement avant trente-cing ans chez I'homme et rarement avant quarante-cing ans chez la femme. Dans sa présentation complète, la maladie hépatique est associée à une fatigue excessive, une mélanodermie, un diabète et des arthropathies. La cirrhose et le carcinome hépatocellulaire en constituent les complications les plus graves.

Le gène responsable a été tout d'abord localisé sur le bras court du chromosome 6 . La stratégie de recherche d'homozygotie par descendance fondée sur I'hypothèse d'un effet fondateur commun a permis d'identifier un gène candidat baptisé HFE-1 qui appartient au complexe majeur d'histocompatibilité de classe I (CMH-1) et au sein duquel une mutation était présente dans $85 \%$ des 356 chromosomes testés contre $3,2 \%$ des 310 chromosomes témoins analysés $\left(\mathrm{m} / \mathrm{s} 1996, \mathrm{n}^{\circ} 11\right.$, p. 1273) [1]. La mutation est une transition du nucléotide 845 (G845A) entraînant la substitution d'une cystéine par une tyrosine en position 282 de la protéine (C282Y). La preuve de la causalité de cette mutation est maintenant bien établie tose génétique est désormais bouleversé par la mise en évidence de cette anomalie qui permet un diagnostic rapide et un dépistage précoce [ 3 , 4]. II est cependant nécessaire de préciser que I'homozygotie pour cette mutation ne suffit pas à rendre compte de la totalité des diagnostics d'hémochromatose génétique mais aussi que sa pénétrance $n$ 'est pas complète, même à un âge avancé. Ce gène code pour une protéine transmembranaire dont la mutation C282Y empêche son expression à la surface cellulaire et ne permet donc pas la formation d'un complexe ternaire avec la $\beta 2$-microglobuline et le récepteur de la transferrine (RTf), interactions nécessaires à la régulation de captage du fer dans plusieurs types cellulaires. Les particularités de la protéine HFE et les conséquences moléculaires de cette mutation ont été décrites dans un précédent numéro de médecine/ sciences $(\mathrm{m} / \mathrm{s}$ $\left.1998, n^{\circ} 4, p .1387\right)$. L'une des fonctions principales de la protéine HFE serait d'opérer une modulation négative de la capture cellulaire du fer via la transferrine (Tf).

En présence de la protéine HFE normale, l'affinité du récepteur pour la transferrine [5] ou le phénomène d'internalisation du complexe RTfTf-Fe [6] seraient diminués, limitant ainsi l'entrée du fer dans la cellule. En I'absence de protéine HFE fonctionnelle, la capture cellulaire du fer deviendrait alors excessive.

Si I'on peut comprendre ainsi que la perte de fonction de la protéine HFE augmente la pénétration du fer dans de nombreuses cellules parenchymateuses (à l'exception des hépatocytes), ce schéma ne permet pas toutefois de rendre compte de l'augmentation de l'absorption intestinale de fer dans le contexte de I'hémochromatose génétique HFE-1. Une seconde mutation, due à une transition du nucléotide 187 (C187G) est responsable de la substitution d'une histidine par un acide aspartique en position 63 de la proteine (H 63D). Des situations équivoques ont été décrites chez des hétérozygotes composites (CY/HD) ou des homozygotes D/ D. En effet, parmi les sujets hétérozygotes composites C282Y/ H63D (CY/HD), un certain nombre présente une surcharge en fer généralement de gravité moindre que la forme classique [7]. La pénétrance de la mutation H63D est de toute façon plus faible que celle de la précédente: I'hémochromatose avérée étant rare chez les homozygotes $D / D$. Cette différence par rapport aux homozygotes C282Y peut s'expliquer par une altération moins sévère de la structure tridimensionnelle de la protéine mutée en position 63. Au contraire de la mutation C282Y, la mutation H63D ne perturbe pas le passage transmembranaire de la protéine HFE. L'acide aspartique apparu en position 63, peut créer un nouveau pont salin compensateur avec la lysine 70 de l'hélice voisine, minimisant alors le retentissement de la mutation H 63D sur la structure tertiaire [8].

Une autre mutation dans l'exon 2, apparue à proximité de la mutation H 63D, entraîne la substitution d'une sérine par une cystéine ( $\mathrm{S} 65 \mathrm{C})$, mais son rôle dans la constitution d'une hémochromatose n'est pas fermement établi $[9,10]$. Aucun homozygote n'a encore été décrit pour cette substitution.

Deux autres mutations pathogènes et uniques pour l'instant ont été aussi rapportées: I'une est une délétion d'une cytosine en position 160 ( $\Delta \mathrm{C}$ 160) [11] qui engendre une protéine tronquée, l'autre, IVS3 $+1 \mathrm{G} \rightarrow \mathrm{T}$, est une mutation qui affecte l'épissage [12]. La mutation $\Delta$ C 160 semble responsable du premier cas décrit d'hémochromatose HFE-1 dominante.

Le gène HFE-1 présente donc pour l'instant la particularité de ne posséder qu'une seule mutation très fréquente dont la pathogénicité est solidement établie chez l'homozygote.

\section{Les autres
hémochromatoses
génétiques}

Il existe dans les populations européennes d'autres hémochromatoses génétiques, dont la liaison au système HLA a été exclue. Une forme juvénile, ou HFE-2, transmise selon le mode autosomique récessif a été caractérisée dans un nombre limité de familles, principalement en I talie, 
pays dans lequel I'hémochromatose HFE-1 ne représente qu'environ $60 \%$ des cas d'hémochromatoses familiales. Les signes cliniques apparaissent entre 14 et 20 ans, ils sont dominés par un tableau d'hypogonadisme d'origine hypophysaire et une insuffisance cardiaque avec ou sans troubles du rythme. La distribution parenchymateuse de la surcharge en fer est équivalente à celle de la forme HFE-1 mais l'accumulation est plus importante et les conséquences déjà graves chez l'adulte jeune. Des études familiales ont permis d'établir une liaison génétique entre I'hémochromatose HFE-2 et une région de $4 \mathrm{cM}$ du bras long du chromosome 1 (1q24) [13] mais le gène n'a pas été encore identifié.

Plus récemment, un phénotype d'hémochromatose familiale a pu être relié à une mutation du récepteur 2 de la transferrine (RTf2) dont le gène est localisé en $7 q 22$, pemettant ainsi d'identifier une nouvelle cause d'hémochromatose: la forme HFE-3. A l'inverse du RTf, le RTf2 est hautement exprimé dans les hépatocytes et agit comme un second récepteur de la transferrine capable de former lui aussi un complexe RTf2-Tf-Fe.

Les sujets atteints, d'origine sicilienne, sont homozygotes pour une transversion $C \rightarrow G$ en position 750 de l'ADNC. Cette mutation fait apparaître un codon stop au niveau du résidu 250 de la protéine (Y250X) [14].

$L$ 'hémochromatose $n$ 'est pas limitée aux populations d'origine européenne puisqu'une forme subsaharienne a été décrite. Sa liaison au locus HLA a été exclue. Dans certaines populations rurales d'Afrique noire, elle peut atteindre jusqu'à $10 \%$ de la population [15]. Cette surcharge, encore dénommée sidérose bantou, résulterait d'une prédisposition génétique, exacerbée par une alimentation riche en fer dont l'origine pourrait être liée à la consommation de bière à partir d'un conditionnement vecteur de fer $[16,17]$. Les hétérozygotes pour le locus lié à la maladie développeraient une surcharge en cas d'ingestion excessive de fer alors que la surcharge des homozygotes serait spontanée [18].

\section{Les thalassémies}

Les thalassémies, maladies autosomiques le plus souvent récessives mais dont plusieurs formes dominantes ont été décrites [19] ont une définition phénotypique simple et précise: une diminution ou une absence totale de biosynthèse d'une ou de plusieurs chaînes de globine. Ces dernières réalisent dans leurs formes graves, voire intermédiaires, de véritables surcharges en fer, aggravées par les traitements transfusionnels, mais préexistants néanmoins à ces derniers [20]. Le degré de gravité des thalassémies dépend non seulement du nombre de gènes globine atteints, mais aussi de la nature de la mutation causale. On distingue les $\beta$ thalassémies qui affectent la synthèse des chaînes $\beta$-globine et qui sont majoritairement la conséquence de mutations ponctuelles, des $\alpha$-thalassémies le plus souvent dues à des délétions. Les formes les plus graves se traduisent par une anémie hémolytique sévère et une érythropoïèse inefficace qui s'accompagnent d'une hypersidérémie, compliquée d'une surcharge hépatique au départ modérée mais aggravée par les traitements transfusionnels. Cependant, même en l'absence de transfusions sanguines, I'hypersidérémie est présente [21]. Ces maladies génétiques, réparties sur le pourtour du bassin méditerranéen, s'étendent au Moyen-Orient et au sub-continent indien, affectant aussi une partie du Sud-Est asiatique et de l'Asie centrale. Elles sont également présentes sur une large portion du continent africain.

Dans I'hémochromatose comme dans les thalassémies, il existe un stockage excessif du fer sans que l'on en comprenne encore bien les mécanismes intimes. L'objectif thérapeutique est la soustraction du fer en excès mais les modalités varient: dans I'hémochromatose génétique, les saignées régulières et contrôlées sont la base du traitement curatif, d'autant plus efficace qu'il est effectué au stade précoce, en tout cas au stade précirrhotique des complications. Dans les thalassémies, à quelques exceptions près, la correction de l'anémie prime, au moins au début, sur la soustraction du fer qui se fera alors par chélation du métal.

\section{Fréquence allélique et repartition géographique de l'hémochromatose génétique HFE-1}

L'hémochromatose génétique HFE-1 est incontestablement, parmi les maladies, monogéniques, celle dont la prévalence est la plus élevée en Europe de l'Ouest où elle affecte environ un sujet sur 300 avec un pic de prévalence en Irlande et en Bretagne où elle peut atteindre respectivement jusqu'à un sujet sur 100 et un sur $180[22,23]$. La fréquence allélique de la mutation C282Y diminue avec la latitude et semble-t-il, selon un gradient Nord-O uest $\rightarrow$ Sud-Est (figure 1). On passe en effet d'un maximum de $14 \%$ dans une population irlandaise à des valeurs comprises entre $7 \%$ et $8 \%$ dans l'Ouest de la France, à $4 \%$ dans l'Est et enfin, à une fréquence voisine de $1 \%$ dans le sud de l'Italie. La fréquence moyenne de l'allèle $Y$ a été récemment établie en Europe où elle est de 6,6\% [24]. Une estimation fondée sur la fréquence des génotypes YY publiés sur plus de 30000 échantillons nous permet d'estimer à environ 1200000 les homozygotes putatifs pour ce génotype dans 15 pays européens. En France, le nombre d'homozygotes est vraisemblablement compris entre 120000 et 160000 soit entre 2 et 3 sujets sur mille. Le nombre d'hétérozygotes est évidemment considérable, puisqu'il atteint environ 27 millions d'individus pour 360 millions d'habitants dans ces mêmes 15 pays; en moyenne un individu sur 13. La mutation H63D est encore plus fréquente, elle atteint $10 \%$ à $20 \%$ en France avec une moyenne en Europe de $14 \%$ [24].

\section{Fréquences alléliques et repartition géographique des thalassémies}

Les sujets originaires des pays ou continents dans lesquels l'hémochromatose HFE-1 est pratiquement 


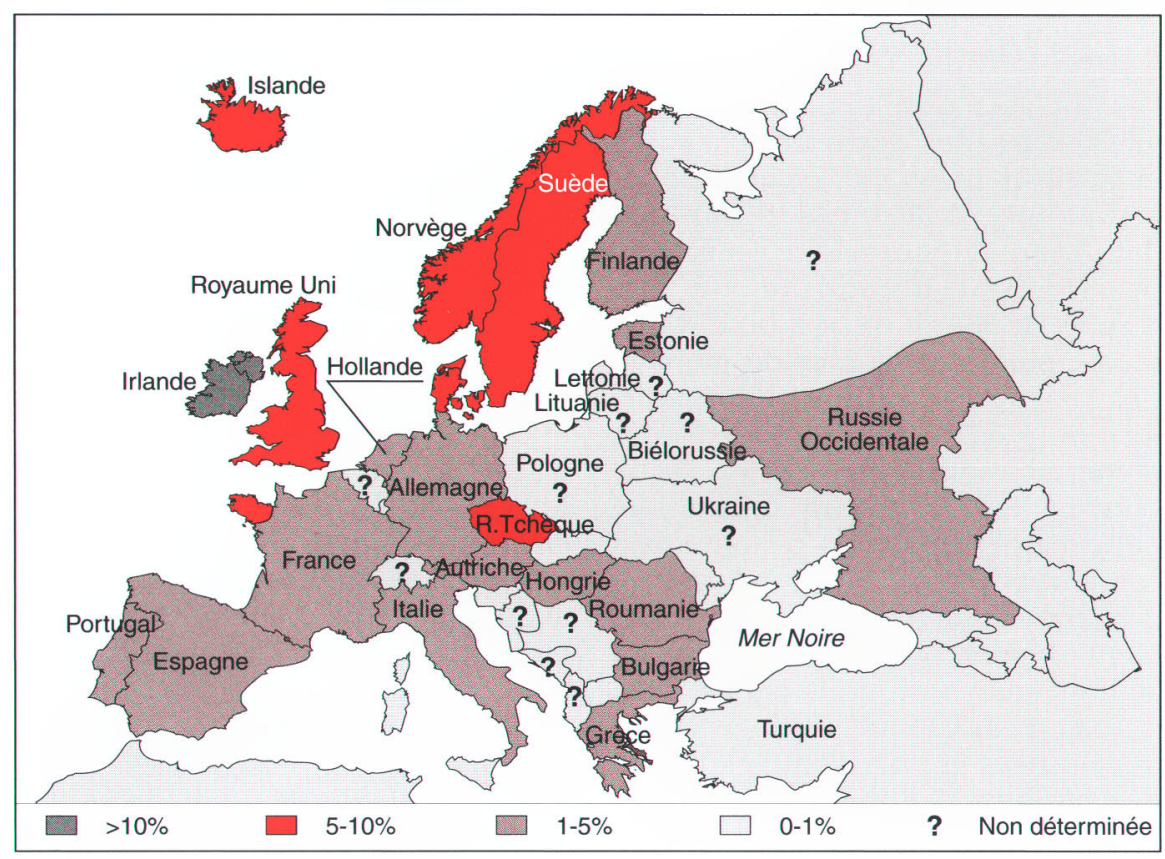

Figure 1. Répartition géographique et ordre de fréquence de la mutation C282Y en Europe (carte établie d'après [22, 24]).

absente ne sont toutefois pas dispensés d'augmenter leur stockage tissulaire en fer. En effet, en Asie, en Afrique ainsi que sur le pourtour sud du bassin méditerranéen où la fréquence de l'allèle $Y$ a été trouvée nulle ou presque nulle [25], les thalassémies prennent le relais de I'hémochromatose génétique contribuant ainsi à étendre sur l'ensemble du globe, le spectre des surcharges en fer. Le nombre approximatif d'hétérozygotes pour un allèle $\beta$-thalassémique dans le monde est estimé à environ 180 millions d'individus et à environ 200 millions, le nombre d'individus porteurs d'un seul allèle $\alpha$-thalassémique [26].

Si I'on porte sur une carte des continents les zones où les fréquences allèliques des mutations responsables de surcharges en fer sont supérieures à 0,01 , il est surprenant de constater que la quasi totalité de la surface du globe est couverte et que là où I'hémochromatose génétique est absente on trouve les thalassémies (figure 2). On peut donc s'interroger sur les raisons

\section{Origine des surcharges en fer}

La loi de Hardy-Weinberg, couramment utilisée en génétique des populations pour calculer des fréquences alléliques, nous apprend qu'à une nouvelle génération, la fréquence est la même qu'à la génération précédente à la condition que cet allèle ne mute pas, que la population considérée soit en équilibre ( $c$ 'est-à-dire que sa taille soit suffisamment grande, qu'il n'existe ni migration, ni sélection et que les croisements $s^{\prime} y$ réalisent de façon aléatoire). Cette loi est surtout intéressante dans sa définition restrictive car elle énumère I'ensemble des facteurs qui peuvent modifier ou faire évoluer les génotypes et donc les populations considérées. Une mutation, à elle seule, ne peut guère faire évoluer une population humaine. Ce sont essentiellement des mécanismes de sélection, de migration ou de dérive génétique qui font évoluer les grands effectifs. La question que nous nous posons est de savoir, parmi ces événe- ments, quel est celui qui, le plus probablement, serait la cause des fréquences alléliques élevées responsables des surcharges en fer héréditaires.

Les événements moteurs de l'évolution des populations humaines ont été considérablement transformés depuis quelques milliers d'années. Le nombre d'individus vivant sur la terre a été multiplié par plus de mille depuis les débuts de l'agriculture (il y a environ 10000 à 12000 ans). L'effet de la dérive génétique s'est donc considérablement estompé au fur et à mesure des millénaires. Depuis environ 2000 à 3000 ans, elle est, pourrait-on dire selon l'expression de Cavalli-Sforza, à peu près «gelée» pour les populations européennes [27].

Un argument majeur plaide en faveur d'un enrichissement important en allèle C282Y par un mécanisme de pression sélective, $c^{\prime}$ est celui de son âge récent comparé à sa fréquence élevée. L'origine de cette mutation remonterait à 40 générations au minimum et à 160 générations au maximum, soit n'importe quand entre 1000 et 4000 ans [28]. La fixation d'une mutation par dérive génétique est un processus extrêmement lent (en moyenne, $1 / 4 \mathrm{~N}$ générations, $\mathrm{N}$ étant la taille effective de la population) et I'on voit mal dans la seule hypothèse d'une dérive génétique comment la mutation C282Y aurait pu se propager à tant d'individus en un nombre de générations si restreint. L'apparition récente de la mutation $\mathrm{C} 282 \mathrm{Y}$ à la suite d'un effet fondateur unique permet de faire I'hypothèse d'un avantage sélectif.

La datation approximative de la mutation C282Y correspond aux dates d'origine probables de la majorité des mutations thalassémiques, comme celle d'ailleurs de la drépanocytose [29]. Les différentes surcharges en fer sont donc des événements relativement récents et, semble-t-il, concomitants.

La mutation $\mathrm{H}$ 63D est d'origine plus ancienne et remonte à environ 7000 à 8000 ans, mais un phénomène dispersif aléatoire paraît également peu probable pour expliquer qu'en moyenne un sujet sur six est hétérozygote pour cette mutation dont 


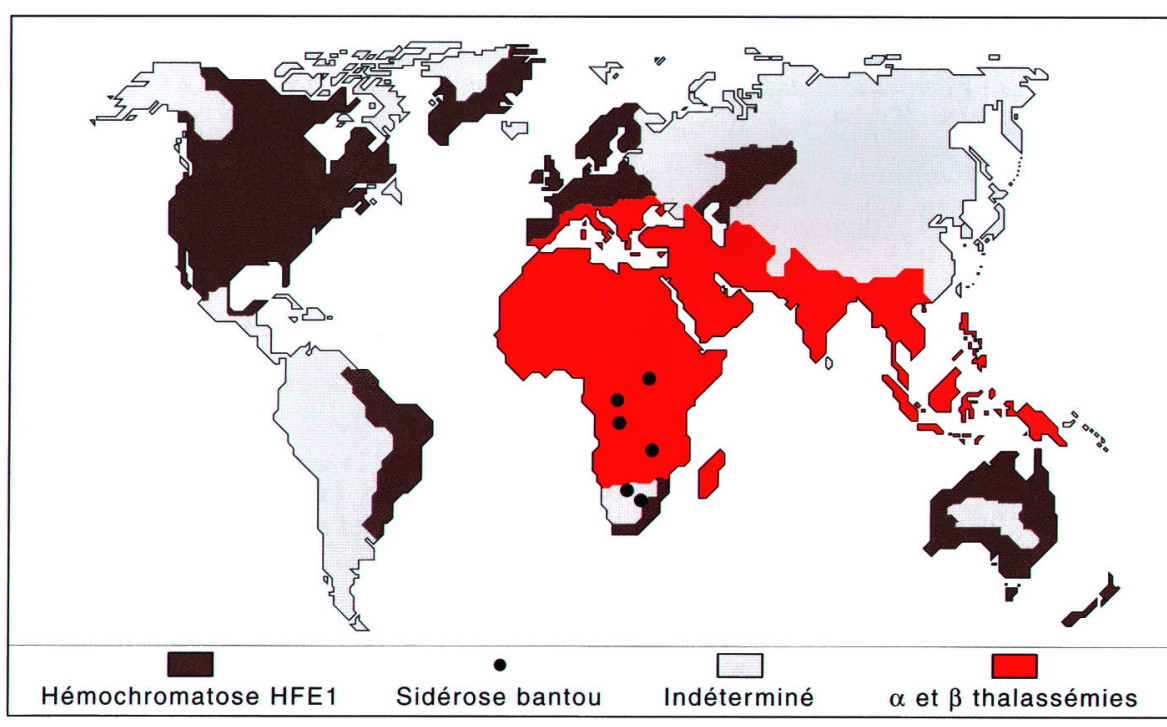

Figure 2. Distribution mondiale des surcharges en fer d'origine génétique dont les fréquences alléliques sont égales ou supérieures à $\mathbf{1 \%}$ (carte établie d'après [20, 22, 24, 26, 29]).

I'origine multicentrique est très vraisemblable [30, 31], comme pour la drépanocytose.

\section{L'hypothèse d'un avantage sélectif sur le métabolisme du fer}

Le mode le plus fréquent de la sélection dite «naturelle» est une action sur les phénotypes dans un contexte global incluant des facteurs environnementaux.

S'il existe une adaptation sélective aux surcharges en fer, on doit montrer que les hétérozygotes ont euxmêmes une surcharge, même modérée, et surtout qu'elle leur procure un certain avantage, où encore que les homozygotes ne sont pas désavantagés sur le plan de la reproduction. Cela est démontré, pour l'hémochromatose HFE-1, grâce d'une part à des souris chez lesquelles la mutagenèse dirigée a permis d'obtenir les trois génotypes pour I'hémochromatose: homozygote normal, hétérozygote et homozygote muté, respectivement $(C / C, C / Y, Y / Y)$. Les données obtenues montrent clairement que les hétérozygotes augmentent leur réserve en fer de facon significative par rapport aux individus normaux [32]. D'autre part, l'apparition habituellement tardive des complications viscérales de la surcharge en fer n'est en aucun cas une barrière à la reproduction chez l'homozygote. On peut signaler que la co-segrégation en trans de la mutation C282Y avec la mutation H63D augmente de façon significative le coefficient de saturation de la transferrine par rapport aux sujets de génotypes $\mathrm{CC} / \mathrm{HH}$, $\mathrm{CC} / \mathrm{HD}, \mathrm{CY} / \mathrm{HH}$ [33].

Bien que la pression sélective exercée par l'agent du paludisme sur les phénotypes thalassémiques soit très probable [34], I'hypothèse d'un second avantage sélectif des thalassémies mineures sur le métabolisme du fer n'est pas non plus déraisonnable.

II semble bien que le schéma de la prime à I'hétérozygote puisse s'appliquer aussi aux thalassémies mineures dans les carences martiales. II est rapporté en effet que la majorité des sujets hétérozygotes pour une $\beta$-thalassémie ou un trait $\alpha$-thalassémique ont un fer sérique relativement augmenté [35]. En tout cas, les carences martiales sont de toute façon plus sévères chez les individus normaux que chez les sujets hétérozygotes [36]. Cela est aussi vrai au cours de la grossesse [37] et la fécondité des femmes hétérozygotes est plus élevée que celle de femmes appartenant à un groupe témoin ne présentant pas de thalassémie [38]. Quant à I'ané- mie des thalassémies mineures, elle est généralement bien tolérée.

La protection assurée par une thalassémie mineure contre les carences martiales semble ainsi réelle, comme l'est encore davantage celle qui est assurée par l'hémochromatose génétique. Cette forte pression de sélection sur le métabolisme du fer aurait ainsi permis une protection contre les carences martiales et, de ce fait, une meilleure résistance à certaines infections et la possibilité de grossesses multiples.

\section{Sélection} par un agent infectieux ?

On sait depuis $\mathrm{H}$ aldane [39] que la mutation unique responsable de la drépanocytose a subi une forte pression de sélection grâce aux plasmodiums, agents des différentes formes de paludisme. Cette hypothèse est étayée par de très nombreuses études épidémiologiques et biologiques, elle est désormais admise par la communauté scientifique. Le même parasite semble aussi impliqué dans une sélection des mutations responsables des thalassémies [40]. Des travaux réalisés dans les îles du sud-ouest du Pacifique ont permis de suspecter une sélection des $\alpha$ thalassémies par l'agent du paludisme. Une réponse immunologique plus rapide des thalassémiques contre le parasite serait le mécanisme biologique sélectionné $[41,42]$. II existe d'autres exemples: la sélection d'une autre maladie génétique, la mucoviscidose, par un agent infectieux a fait récemment I'objet d'un travail remarquable. II a été démontré que Salmonella typhi, bactérie responsable de la typhoïde, utilise la protéine CFTR pour pénétrer dans les cellules épithéliales de I'intestin. La pénétration est considérablement réduite chez I'hétérozygote et absente chez l'homozygote muté [43].

La résistance à un agent infectieux pourrait donc être un mécanisme sélectif assez général de nombreuses maladies génétiques. La sélection de la protéine HFE mutée par un agent infectieux pourrait répondre à un schéma analogue.

En effet, la mutation C282Y, ne permet pas à cette protéine d'atteindre 
la surface cellulaire et abolirait donc toute interaction avec une bactérie, un parasite ou un virus.

$L a$ région de la protéine HFE qui pourrait être impliquée dans un mécanisme de reconnaissance et/ ou de pénétration d'un agent infectieux, pourrait être localisée dans l'exon 3 qui code pour le domaine $\alpha 1$, dans lequel se situe la mutation H63D. Cette dernière mutation aurait donc aussi un effet délétère sur la reconnaissance d'un agent infectieux [25] . La nature de l'agent pathogène reste cependant encore à préciser et un mécanisme de sélection mono- ou multi-factoriel ne peut-être rejeté. Plusieurs arguments plaident donc en faveur d'un processus d'adaptation de ces surcharges en fer à des conditions d'environnement. Pour ce qui concerne les thalassémies, on comprend bien la valeur sélective supérieure de I'hétérozygote sur I'homozygote. Dans le cas de l'hémochromatose, la sélection semble encore plus bénéfique: les homozygotes mutés n'étaient pas désavantagés dans le passé, car cette maladie ne pouvait être un inconvénient à un âge que les êtres humains n'atteignaient pas en moyenne il y a deux ou trois mille ans. Aujourd'hui, il en va tout autrement, les «génotypes rouillés» sont lourds du fardeau métallique qu'ils peuvent stocker

\section{Remerciements}

Les auteurs remercient Jacques Ninio et Jean Pierre Capron pour la lecture critique du manuscrit ainsi que Marie Chesneau pour le travail de cartographie.

Une partie des travaux résumés dans cet article ont été financés par le Consortium Européen de Recherche sur I'Hémochromatose (QLT 1999-02237) et le pôle GBM-PE (Jean-Pierre Libert).

\section{RÉFÉRENCES}

1. Feder JN, Gnirke A, Thomas W, et al. A novel $\mathrm{MHC}$ class like gene is mutated in patients with hereditary haemochromatosis.
2. Zhou XY, Tomatsu S, Fleming RE, et al. HFE gene knockout produces mouse model of hereditary hemochromatosis. Proc Natl A cad Sci USA 1998 ; 95 : 2492-7.

3. Rochette J, Capron D, Capron JP, Julier $C$. Screening for hereditary hemochromatosis. A m J Gastroenterol 2000; 95 : 1368-9.

4. The European $\mathrm{H}$ aemochromatosis Consortium. Diagnosis and management of haemochromatosis since the discovery of the H FE gene: a European experience. $\mathrm{Br}$ J $\mathrm{H}$ aematol 2000; 108: 31-9.

5. Lebron JA, West AP, Bjorkman PJ. The hemochromatosis protein HFE competes with transferring for binding to the transferrin receptor. J M ol Biol 1999; 294: 23945.

6. Salter-Cid L, Brunmark A, Li Y, et al. Transferrin receptor is negatively modulated by the hemochromatosis protein HFE: implications for cellular iron homeostasis. Proc Natl Acad Sci USA 1999; 96: 5434-9.

7. Moirand $R$, Jouanolle $A$, Brissot $P$, Le Gall J, David V, Deugnier Y. Phenotypic expression of the HFE mutations: a French study of 1110 unrelated iron-overloaded and relatives. Gastroenterology 1999; 116: 372-7.

8. Dupradeau FY, Altenberg-Greulich B, Warin R, Fuentes V, M onti JP, Rochette J. A 3-dimensional model building by homology of the HFE protein: molecular consequences and application to antibody development. Biochem Biophys Acta 2000; 1481: 213-21.

9. Douabain V, Moirand $R$, Jouanolle $A$, et al. Polymorphisms in the $\mathrm{HFE}$ gene. $\mathrm{H}$ um H ered 1999; 49: 21-6.

10. Mura C, Raguenes O, Ferec C. HFE mutations analysis in 711 haemochromatosis probands: evidence for $\mathrm{S65C}$ implication in mild form of hemochromatosis. Blood 1999; 93: 2502-5.

11. Pointon JJ, Shearman AT, Merryweather-Clarke A, Robson KJH. A single nucleotide deletion in the putative haemochromatosis gene in a patient who is negative for both the $\mathrm{C} 282 \mathrm{Y}$ and $\mathrm{H} 63 \mathrm{D}$ mutations. International Symposium Iron in Biology and Medicine. Saint-Malo, France, 16-20 juin 1997, p. 268.

12. Wallace DF, Dooley JS, Walker AP. A novel mutation of HFE explains the classical phenotype of genetic hemoch romatosis in a C282Y heterozygote. Gastroen terology 1999; 116: 1409-12.

13. Camaschella $C$, Roetto $A$, Cicilano $M$, et al. Juvenile and adult hemochromatosis are distinct genetic disorders. Eur J Hum Genet 1997 ; 5 : 371-5.

14. Camaschella $C$, Roetto $A$, Cali $A$, et al. The gene TFR 2 is mutated in a new type of haemochromatosis mapping to $7 q 22$. N at Genet 2000; $25: 14-5$.
15. Gordeuk VR. Hereditary and nutritional iron overload. Baillieres Clin $\mathrm{H}$ aematol 1992; 5: 169-86.

16. Gordeuk VR, Mukiibi J, Hasstedt SJ. Iron overload in Africa: interaction between a gene and dietary iron content. $\mathrm{N}$ Engl J M ed 1992; 326: 95-100.

17. Moyo VM, Mandishona $E, H$ asstedt SJ, et al. Evidence of genetic transmission in African iron overload. Blood 1998; 91: 1076-82.

18. Andrews N. Disorders of iron metabolism. N Eng J M ed 1999; 341: 1986-95.

19. Thein SL, Hesketh C, Taylor $P$, et al. Molecular basis for dominantly inherited inclusion body $\beta$-thalassemia. Proc Natl Acad Sci USA 1990; 87: 3924-8.

20. Weatherall DJ, Clegg JB. The thalassaemia syndromes, $4^{\mathrm{e}}$ ed. Oxford: Blackwell Scientific, 2001 (sous presse).

21. Thein SL, Rochette J. Disorders of hemoglobin structure and synthesis. In: Jameson JL, ed. Principles of molecular medicine. Totowa: H umana Press, 1998: 179-90.

22. Merryweather-Clarke AT, Pointon JJ, Shearman JD, Robson KJH. Global prevalence of putative haemochromatosis mutations. J M ed Genet 1997; 34 : 275-8.

23. Jezequel $P$, Bargain $M$, Lellouche $F$, Geffroy $F, D$ orval $I$. Allele frequencies of hereditary hemochromatosis gene mutations in a local population of west Brittany. $\mathrm{H}$ um Gen et 1998; 102: 332-3.

24. Merryweather-Clarke AT, Pointon JJ, Jouanolle AM, Rochette J, Robson KJH'. Geography of HFE C282Y and H63D mutations. Genet Test 2000; 4: 183-98.

25. Roth $M$, Giraldo $P$, Hariti $G$, et al. Absence of the hemochromatosis gene Cys282Tyr mutation in three ethnic groups from Algeria $5 \mathrm{Mzb}$ ), Ethiopia, and Senegal. Immunogenetics 1997; 46: 222-5.

26. Tribout BA, Rees DC, Webster MT, et al. Clotting in Europe, iron overload all over the world. Blood 1998; 92 suppl.1: 45a.

27. Cavalli-Sforza LL. Genes, people and Ianguages, New York: North Point Press, 2000 : 205-6.

28. Thomas W, Fullan A, Loeb DB, McClelland $E E, B a c o n ~ B R$, Wolff RK. A haplotype and linkage disequilibrium analysis of the hereditary hemochromatosis gene region. H um Genet 1998; 102: 517-25.

29. Embury SH, Hebbel RP, Mohandas N, Steinberg MH. Sickle cell disease: basic principles and clinical practice. New York: Raven Press, 1994: 95-7.

30. Rochette J, Pointon JJ, Fisher CA, et al. Multicentric origin of hemochromatosis gene (HFE) mutations. Am J Hum Genet 1999 ; 64 : 1056-62.

31. Pagnier J, Mears JG, Dunda-Belkhodja 0 , et al. Evidence for the multicentric origin of the sickle cell hemoglobin gene in Africa. Proc Natl Acad Sci USA 1984; 81: 1771-3. 


\section{RÉFÉRENCES}

32. Levy JE, Montross LK, Cohen DE, Fleming MD, Andrews NC. The C282Y mutation causing hereditary hemochromatosis does not produce a null allele. Blood 1999; 94: $9-11$.

33. Cadet $E$, Dautreaux-Lagarde $M$, Perez AS, et al. Unexpected high prevalence of compound heterozygotes for C282Y and H63D mutations in a normal population from North of France. Blood 2000;96 (suppl) : 3666.

34. Flint J, Hill, AVS, Bowden DK, et al. $H$ igh frequencies of $\alpha$-thalassaemia are the result of natural selection by malaria. Nature 1986 ; 321 : 744-9.

\section{Summary}

The rusty genotypes

Two main genetic entities lead to iron overload: hemochromatosis and thalassemia. Genetic hemochromatosis HFE-1 type is a common autosomal recessive disease affecting about $1 / 300$ individuals of european descent. HFE-1 type hemochromatosis is associated with the C282Y mutation of the HFE gene, and the H63D mutation to a much lesser degree. Allele frequency of the C282Y type is about $6,6 \%$ in Europe and the average of the $\mathrm{H}$ 63D allele frequency is about $13,4 \%$. Thalassemia is prevalent in a wide belt of countries extending from the mediterranean basin down to Africa and through the Middle East, the indian subcontinent and into south east Asia and China. Population genetic studies show that thalassemias are common where primary hemochromatosis is not. There is little reproductive disavantage to $\mathrm{C} 282 \mathrm{Y}$ homozygotes and even less to heterozygotes. No major disvantage appears for the H63D mutation. Our results suggest that there is or has been selection pressure favoring these mutations making genetic drift an unlikely explanation.
35. White JM, Richards R, Jelenski G, Byrne $M$, Ali $M$. Iron state in alpha and beta thalassaemia trait. J Clin Pathol 1986; 39: 256-9.

36. Galanello R, Turco MP, Barella $S$, et al. Iron stores and iron deficiency anemia in children heterozygous for beta-thalassemia. $\mathrm{H}$ a ematologica $1990 ; 75$ : 319-22.

37. Saraya AK, Kumar R, Choudhry VP, Kailash S, Sehgal AK. A study of serum ferritin in beta thalassemia. Iron deficiency and overload. Am J Clin Pathol 1985; 84: 103-7.

38. Astolfi P, Degioanni A, Tagarelli A, Zei $G$. Past malaria, thalassemia, and woman fertility in southern Italy. Ann Hum Biol 1999; 26: 163-73.

Until now it has been assumed that the selective advantage conferred by HFE mutations was the prevention of iron deficiency; this would include protection against anemia due to hookworm infestation, multiples pregnancies, a diet lacking in iron or any combination of these factors. However if this were solely the case, then one might expect that one more of the HFE mutations would have reached fixation in countries where there are high levels of anemia; this has not been observed yet. Because of wild-type HFE protein is expressed at the cell surface, one could speculate that this protein is the receptor for some infectious agents. The region of the protein that might be involved could be the exon 3 which encodes the $\alpha 1$ domain where the H63D mutation is found. The C282Y mutation does not allow the HFE-1 protein to reach the cell surface. Like thalassemia, hemochromatosis could be the result of a selection pressure involving resistance to infectious agent. By now, the entire world population carries an unfortunate genetic burden leading to iron overload.
39. Haldane JBS. The rate of mutation of human genes. $8^{\text {th }}$ International Congress of Genetics. Oxford, 1949 (suppl. 35) : 267-73.

40. Clegg JB, Weatherall DJ. Thalassemia and malaria: new insights into an old problem. Proc Assoc Am Phys 1999; 111: 278-82.

41. Weatherall DJ. Host genetics and infectious diseases. Parasitology 1996; 112 (suppl) : S23-9.

42. Weatherall DJ, Clegg JB. Genetics disorders of hemoglobin. Semin H ematol 1999; 36 : 24-37.

43. Pier GB, Grout M, Zaidi T, et al. Salmonella typhi uses CFTR to enter intestinal epithelial cells. Nature 1998; 393: 79-82.

\section{Estelle Cadet \\ Renaud Warin Anne-Sophie Perez Jacques Rochette}

UPRES-EA 2629 et Service de génétique moléculaire médicale, UPJV, Faculté de médecine, 3, rue des Louvels, 80036 Amiens, France.

\section{Dominique Capron}

Service d'hépato-gastro-entérologie, CH U d'Amiens, place V.-Pauchet, 80054 Amiens, France.

\section{TIRÉS À PART}

J. Rochette. 